\title{
A Study of Surfactant Effects on the Liquid-Phase Motion around a Zigzagging-ascent Bubble Using a Recursive Cross Correlation PIV
}

\author{
Saito, T.*, Sakakibara, K.**, Miyamoto, Y.** and Yamada, M.** \\ * Graduate School of Science and Technology, Shizuoka University \\ 3-5-1 Johoku, Naka-ku, Hamamatsu, Shizuoka 432-8561, Japan \\ Corresponding author, ttsaito@ipc.shizuoka.ac.jp \\ **Faculty of Engineering, Shizuoka University \\ 3-5-1 Johoku, Naka-ku, Hamamatsu, Shizuoka 432-8561, Japan
}

ABSTRACT

The velocity field around a zigzagging ascent single bubble has a wide dynamic range. It is hard to precisely measure such velocity field via conventional PIV. In this study, to overcome this difficulty the authors employed recursive cross-correlation algorithm and a high-speed video camera. The analysis code of a newly developed PIV system showed a high performance in measuring velocities of a wide dynamic range. At first, the authors demonstrate the performance of the recursive cross-correlation PIV algorithm as compared with the results obtained by conventional PIV algorithm using the standard PIV images with a wide dynamic range presented by The Visualization Society of Japan. At second, the system was applied to the simultaneous measurement of the surrounding liquid motion (i.e. liquid-phase velocities) and surface motion of a single bubble zigzagging upward in purified water and contaminated water. At third, surfactant effects on the surrounding liquid motion (i.e. intensity of the vorticity, size of the vorticity and the structure of a hairpin-like vortex) were discussed as well as those on centre-of-gravity and surface motions of the bubble. In this study, a very small amount of surfactant (1-pentanol) solutions were examined. Although the bulk surface tension was almost the same as that of purified water, the bubble motion and the surrounding liquid motion in the solutions (i.e. contaminated system) were very different from those in purified water (i.e. purified system). A critical concentration of a surfactant was found out. At the critical concentration, the intensity and size of the vorticity become the largest. From the consideration of coupling the asymmetric surface motion of the bubble and the surrounding liquid motion, it was found out that the Marangoni stress owing to a concentration gradient of the adsorbed surfactant on the bubble surface plays a great role in both bubble and surrounding liquid motion.

Keywords PIV, recursive cross correlation algorithm, surfactant effect, bubble, liquid-phase motion, Marangoni effect 


\section{INTRODUCTION}

Gas-liquid two-phase flows are important and frequently encountered in various industrial plants such as chemical plants, bio reactors, environmental plants, nuclear reactors and so on. The major characteristics of the flows are of buoyancy driven flows with dispersed free surfaces, and interaction between the bubbles and their surrounding liquid. The flows have multi-scale ranging phenomena from molecular scale to continuum scale. In the above, the interaction of single bubble motion and its surrounding liquid motion is the smallest scale from a viewpoint of bubble number density. In order to improve the industrial plants with gas-liquid two-phase flows, deep understanding of the complex flows is essential. The liquid phases of the flows in the industrial plants are usually non-purified system; therefore the influences of the bubble-surface contamination should be clarified from a practical viewpoint.

Fan and Tsuchiya (1990) systematically carried out experiments on bubble-surface motion under some contaminated systems, and proposed the relation between the surface oscillation and contamination concentration. Takagi et al. (2003) discussed influences of a surface active surfactant on the motion of a spherical bubble less than $1 \mathrm{~mm}$ experimentally and numerically; they described that the bubble rising velocity is largely reduced by Marangoni convection induced by the surface active surfactant adsorbed on the bubble surface. Initial bubble-shape deformation occurring at a moment of launching a bubble affects its subsequent trajectory and terminal velocity, extremely (Wu and Gharib 2002; Tomiyama et al. 2002). Tomiyama (2002) described that a bubble in a diameter range at which the surface tension is dominant and of small initial shape deformation shows similar behaviour to that in a contaminated system. Furthermore, Duineveld (1995) elucidated the relation between rising motion and surface motion of a single bubble in a pure and rest water. Fujiwara et al. (2004) discussed shape deformation and flow structure of a bubble held under a small mesh using PIV/LIF. Although many researches on bubble behaviour in both purified and contaminated systems were reported, the dynamics of a liquid phase around a bubble and understanding of surfactant effect based on it are still exceedingly insufficient.

The velocity field around a zigzagging ascent bubble has a wide dynamic range. It is hard to precisely measure such velocity field via conventional type of PIV (Adrian 1991). In this study, to overcome this difficulty the authors employed recursive cross-correlation algorithm (Westerweel, J. et al. 1997; Hart 1999) and a high-speed video camera. The analysis code of a newly developed PIV system showed high performance in measuring velocities of a wide dynamic range. At first, the authors demonstrate the performance of the recursive cross-correlation PIV algorithm as compared with the results obtained by conventional type of PIV algorithm using the standard PIV images with a wide dynamic range presented by The Visualization Society of Japan. At second, the developed system is applied to simultaneous measurement of the velocity field around a zigzagging ascent bubble and its surface motion. At third, surfactant effects on the surrounding liquid motion (i.e. intensity of the vorticity, size of the vorticity and the structure of a hairpin-like vortex) are discussed as well as those on centre-of-gravity and surface motion of the bubble. In this study, a very small amount of surfactant (1-pentanol) solutions were examined. 
Although the bulk surface tension is the same as that of purified water, the bubble motion and the surrounding liquid motion in the solutions (i.e. contaminated system) are very different from those in purified water (i.e. purified system). A critical concentration of a surfactant was found out. At the critical concentration, the intensity and size of the vorticity becomes the largest. From the consideration coupling the asymmetric surface motion of the bubble and the surrounding liquid motion, it has been found out that the Marangoni stress owing to a concentration gradient of the adsorbed surfactant on the bubble surface plays a great role in the bubble motion in the contaminated system.

\section{EXPERIMENTAL SETUP AND ANALYSIS}

\subsection{Experimental setup}

Figure 1 shows an experimental setup employed in the present study. An acrylic water vessel (a) (150 $\mathrm{mm} \times 150$ $\mathrm{mm} \times 500 \mathrm{~mm}$ ) was filled with purified water (tap water filtrated by $1 \mu \mathrm{m}$ filter, ion-exchanged and degassed) to a depth of $450 \mathrm{~mm}$. An isolated single bubble was launched from a hypodermic needle with $0.4 \mathrm{~mm}$ in diameter fixed on the bottom of the acrylic vessel under precise control of the flow rate and pressure of supplied air from a gas cylinder, using a bubble launch device illustrated in Figure 2. This device precisely and repeatedly formed isolated uniform bubbles at controllable launching timing. An audio speaker pushed the air which was sealed in a clearance between the speaker cone and acrylic box, under a well controlled pressure and flow; it formed a bubble uniform in diameter and initial shape at the hypodermic needle. A check valve was mounted in order to prevent the back-flow induced by oscillation of the cone. The bubble launch timing was arbitrarily controlled by the function generator; in the present experiments, it was set at 4 [sec]. Extremely high reproducibility in size, ascent trajectory and surface motion of bubbles was achieved by using the hypodermic needle and controlling the flow rate and pressure of air adequately (Miyamoto \& Saito, 2005). The average diameter of the bubbles was $2.89 \mathrm{~mm}$ in equivalent diameter (in purified system). In addition, it was confirmed in advance that the wakes induced by the former bubble did not affect the motion of the later bubble launched after the interval.

The beam from a laser sensor (c) intersected with the zigzag trajectory of the bubbles, and detected the bubble passing. The TTL signal from the laser sensor was inputted to a function generator. Digital recording of a high-speed video camera (Vision Research Corporation, Phantom V9.0) (d) was triggered by functional pulse wave from the function generator; exposure time: $400 \mu \mathrm{s}$, frame rate: $1900 \mathrm{fps}$, number of pixels: $960 \times 960$ pixels, spatial resolution: $13.75 \mu \mathrm{m} /$ pixel. Surface and centre-of-gravity motions of the isolated single bubbles were filmed using ring-array red LED (e) (wavelength: 660nm). Simultaneously, LIF method was employed to visualize its surrounding liquid motion; LIF particles were of $50 \mu \mathrm{m}$ in diameter, $532 \mu \mathrm{m}$ in excitation wavelength and $570 \mathrm{~nm}$ in emission wavelength. A Light source of PIV was a green laser (f) (YVO4-YAG, 2W, 532nm). The laser beam was split into two directions by half mirror (g); two sheet-like laser beams through rod lenses (h) illuminated a measurement region from both sides of the vessel, and uniform light quantity was achieved around the bubble. In 
addition, a concentration of the LIF particles was decided very carefully as keeping free from the effects of the LIF particles on both surface motion and centre-of-gravity motions of the single bubbles; hence the number density of the LIF particles was small in the present study.

Diameters of the single bubbles were calculated from images filmed by another high-resolution video camera $(1024 \times 1024$ pixels $)$ just after released from the needle; in the region of linearly rising trajectory, the bubbles were of an oblate spheroid, and their volume was calculated from the snapshots correctly.

\subsection{PIV algorism}

In PIV measurement, adequate frame rate, frame size and light intensity corresponding to a target flow are required. Furthermore, in PIV analysis, the most appropriate algorism should be selected corresponding to a dynamic range of the target flow and number density of seeding particles. Usually, higher resolution in space is calculated from smaller interrogation area, because the velocity is obtained from a displacement of particles between a pair of interrogation areas. However, an excessively small interrogation area increases the number of incorrect vectors due to decrease of accuracy of pattern matching. On the other hand, an excessively large interrogation area increases accuracy of pattern matching (i.e. a decrease in the number of incorrect vectors); however the spatial resolution descends inevitably, and one can not extract the characteristic structure of a target flow. Hence, it is essential to configure an appropriate interrogation area corresponding to the number density of PIV particles and the fluid motion. The recursive cross-correlation method was adopted as PIV algorithm in the present study. This method is especially effective against a velocity field with a wide dynamic range such as the surrounding liquid motion of a bubble. The authors newly coded recursive cross-correlation PIV, and verified it by analysing the standard PIV images presented by The Visualization Society of Japan.

Figures 3 (a) and (b) are a set of the standard PIV images (256 $\times 256$ pixels). The authors compared the results obtained by recursive cross-correlation PIV with those by conventional cross-correlation PIV algorism.

In the conventional cross-correlation PIV, velocities were calculated as follows (see Figure 4):

i) Setting of brightness distribution functions, $f(x, y)$ and $g(x, y)$ of interrogation areas of $64 \times 64$ (or $32 \times 32$ ) pixels at $\mathrm{t} 1$ and $t_{1}+\Delta t$,

ii) Calculation of power spectra $F(\xi, \eta)$ and $G(\xi, \eta)$ by FFT,

iii) Calculation of cross spectrum by convolution of $F(\xi, \eta)$ and $G(\xi, \eta)$,

iv) Calculation of a cross-correlation coefficient $C_{c c}(x, y)$ by IFFT,

v) Calculation of a displacement $(\Delta x, \Delta y)$ from the peak value in the table of $C_{c c}(x, y)$,

vi) Calculation of a velocity of the interrogation area from the displacement $(\Delta x, \Delta y)$ and time interval $\Delta t$.

The peak value of $C_{c c}(x, y)$ should be close to unity, and signal intensity $\left(1-1 / S_{N R}\right)$ should be large, where $S_{N R}$ represent the ratio of the first peak value to the second one.

In the recursive cross-correlation PIV, velocities were calculated as follows (see Figure 5): 
i) Setting of brightness distribution functions, $f_{l}(x, y)$ and $g_{l}(x, y)$ of initial interrogation areas of $64 \times 64$ (or $32 \times 32$ ) pixels at $t_{l}$ and $t_{l}+\Delta t$,

ii) Calculating power spectra $F_{l}(\xi, \eta)$ and $G_{l}(\xi, \eta)$ by FFT,

iii) Calculating a cross spectrum by convolution of $F_{l}(\xi, \eta)$ and $G_{l}(\xi, \eta)$,

iv) Calculating a cross-correlation coefficient $C_{c c l}(x, y)$ by IFFT,

v) Calculating a displacement $\left(\Delta x_{1}, \Delta y_{l}\right)$ from the peak value in the table of $C_{c c l}(x, y)$,

vi) Calculating the first-phase velocity of the interrogation area from the displacement $\left(\Delta x_{1}, \Delta y_{l}\right)$ and time interval $\Delta t$,

vii) Setting of the second-phase interrogation area of $64 \times 64$ (or $32 \times 32$ ) pixels by "window offset" at $t_{l}+\Delta t$ with reference to the first-phase velocity,

viii) Downsizing the second-phase interrogation area to four sub-interrogation areas of $32 \times 32$ (or $16 \times 16$ ) pixels,

ix) Iterating the processes from ii) to viii) until $8 \times 8$ pixels (or minimum interrogation size). The size of a final interrogation area depends on number density of PIV particles and a target flow.

\subsection{Verification of newly coded recursive PIV, and comparison of results obtained by conventional and} recursive PIV

Figure 6 shows the results on velocity profiles calculated by newly coded recursive PIV at each phase from the first phase $(64 \times 64$ pixels $)$ to the final phase $(8 \times 8$ pixels $)$. The spatial resolution increases gradually with progression of the recursive process; accordingly, the detailed structure of a flow is becoming clearer step by step. Comparisons of velocity vectors, $C_{C C}$ and signal intensity $\left(1-1 / S_{N R}\right)$ between a conventional PIV and recursive PIV are shown in Figures 7 (a) - (d), provided that the size of a final interrogation area is $16 \times 16$ pixels. The velocity vectors in (b) and (d) (: recursive PIV) are remarkably improved as compared with those in (a) and (c) (: conventional PIV). In (a) and (c), the number of incorrect vectors is large. Furthermore, in (b) $C_{C C}$ takes almost unity at large portion of the visualization area; on the other hand in (a), it takes 0.5 or less at large portion. The recursive method significantly improves $C_{C C}$ all over the measurement area. In (d) signal intensity takes 0.6 or more at large portion; meanwhile in (c), it takes 0.45 or less at large portion. Thus, the recursive method is very effective in improving signal intensity (Sakakibara, et al. 2006).

Figure 8 shows a comparison of velocity field between true values (presented by The Visualization Society of Japan) and measured values via newly coded recursive PIV. Although the artificial flow has very wide dynamic range, the measured values extremely correspond with the true values in the entire area except a region marked by red rectangle. From these, one can verify validity of the code and effectiveness of a recursive PIV.

\subsection{Pre-process for PIV and bubble motion analysis}


In order to achieve simultaneous measurement of the bubble motion and its surrounding liquid motion, the authors filmed the bubble and PIV particles via one high-speed video camera. It was needed to separate the PIV particles image from the bubble image. First, an original image (Figure 9 (a)) was processed by median filter to remove noises: a secondary image. Second, a bubble image was extracted by a sequential process of binary image processing, area-threshold processing and grayscale inversion (Figure 9 (c)). Third, a PIV-particle image was obtained by removing the bubble image from the secondary image. Furthermore, the pixels which the bubble occupied were superimposed on luminance value of the peak value of its surrounding area; thus a final image for PIV analysis was obtained (Figure 9 (b)).

Recursive cross-correlation PIV described above was employed; the initial size of an interrogation area was $64 \times 64$ pixels, and the final size was $32 \times 32$ pixels. The coordinates of a bubble contour were obtained from image processing, and the coordinates of centre-of-gravity of the bubble were calculated from them. Furthermore, the coordinates of the bubble contour were transformed to a system of polar coordinates $(r, \theta)$ with their origin at the centre of the bubble. The bubble contour was expressed as follows (Duineveld 1995):

$$
r(\theta)=A_{0}+\sum_{n=1}^{N}\left(A_{n} \cos n \theta+B_{n} \sin n \theta\right)
$$

$A_{n}$ and $B_{n}$ were calculated by FFT with mode order $N$ of 8 . A major diameter $D_{l}$ of the bubble image was calculated as the maximum value (i.e. $\left.D_{l}\right|_{\theta=\theta_{l}}$ ) of $r(\theta)+r(\theta+\pi)$; minor diameter $D_{2}$ was calculated as $r\left(\theta_{l}+\pi / 2\right)+r\left(\theta_{l}+3 \pi / 2\right)$. Aspect ratio was calculated by $D_{2} / D_{l}$. Aspect ratio has been used in many former papers (e.g. Ellingsen and Risso, 2001), however the ratio is exceedingly insufficient in order to discuss shape asymmetry of a bubble. Hence, in the present study, curvature $\left.\kappa(\theta)\right|_{\theta=\theta_{1} \text { or } \theta_{1}+\pi}$ at right and left edges of a bubble was introduced in order to quantitatively discuss the shape asymmetry.

$$
\kappa(\theta)=\frac{\left|r^{2}+2\left(\frac{d r}{d \theta}\right)^{2}-r \frac{d^{2} r}{d \theta^{2}}\right|}{\left[r^{2}+\left(\frac{d r}{d \theta}\right)^{2}\right]^{\frac{2}{3}}}, \text { here } \theta=\theta_{1} \text { or } \theta_{l}+\pi
$$

In addition, dimensionless curvature $\left(\kappa_{R}\right.$ and $\left.\kappa_{L}\right)$ at right and left edges is defined as follows:

$$
\begin{aligned}
& \kappa_{R}=\kappa\left(\theta_{1}\right) D_{e q}, \\
& \kappa_{L}=\kappa\left(\theta_{1}+\pi\right) D_{e q},
\end{aligned}
$$

here $D_{e q}$ represents an equivalent diameter of the bubble. 


\section{RESULTS AND DISCUSSION}

\subsection{Bubble properties}

In the present study, a very small amount of 1-pentanol (150 ppm and $500 \mathrm{ppm})$ was added to the purified water, for the particular purpose of grasping influences of the surface active surfactant on the bubble motion. The bubble properties examined in this experiment are listed in Table 2. As shown in this table, a change in surface tension was very small; therefore Eötvös Number takes almost a constant value.

\subsection{Reproducibility of a bubble shape, orientation and trajectory}

In order to discuss the structure of the surrounding liquid motion of a bubble, high reproducibility of bubble formation, launch and trajectory should be verified; for this specific purpose, the authors controlled the initial shape deformation of a bubble by utilizing a wedge-shape edge of the hypodermic needle, and accurate control of a very small amount of gas flow rate and pressure; furthermore, by utilizing the relation between the initial bubble shape deformation and the subsequent bubble trajectory (Tomiyama, et al., 2002). Figure 10 shows superimposed 100 snapshots of 100 bubbles of diameter range within $\pm 5 \%$ of the average diameter filmed at the same position. The counters of the bubbles coincide mutually; i.e. very high reproducibility of surface motion, shape, orientation and trajectory of the bubbles was simultaneously achieved. Therefore, PIV results of the surrounding liquid motion of the bubbles at the same position and diameter are considered to have the same property statistically.

\subsection{Calculation of vorticity}

Vorticity is very effectual to consider vortex shedding closely linked to zigzagging motion of a bubble. The authors calculated the vorticity by the following equation on the basis of Stokes's theorem:

$$
\omega=\frac{\Gamma}{\Delta X \Delta Z}
$$

where $\omega$ is vorticity; $\Gamma$ is circulation; $\Delta X$ and $\Delta Z$ are an interval of velocity point of PIV. $\Gamma$ was calculated using eight data sets of the PIV results around the objective point. When the data points are positioned inside the bubble, $\omega$ and $\Gamma$ were not calculated.

\subsection{Centre-of-gravity motion and surface motion of a Bubble}


Figure 11 shows bubble trajectory, bubble composition velocity $V$ and aspect ratio $A s$ at 1-pentanol concentrations of 0 (purified water), $150 \mathrm{ppm}$ and $500 \mathrm{ppm}$. It is observed that both centre-of-gravity motion and surface motion of the bubble are affected even by a very small amount of surface active surfactant. The amplitude and period of the bubble trajectory, the average and oscillation of the bubble composition velocity, and the average and oscillation of the aspect ratio changed with increases in 1-pentanol concentration. The average As approached unity with increase in concentration; this correlates closely with dynamic pressure acting on the bubble. The bubble trajectory took the largest amplitude and the longest period at a 1-pentanol concentration of round $150 \mathrm{ppm}$. The average of $V$ took the largest value at purified water. A high-frequency oscillation of $V$ and $A s$ decreased with increase in 1-pentanol concentration; at purified water, $V$ oscillated twitchily; while low-frequency oscillation developed with increase in 1-pentanol concentration, and at $500 \mathrm{ppm}$ the high-frequency oscillation disappeared. A period of the low-frequency oscillation was twice the period of the zigzagging motion. Fluctuation of $V$ correlated closely with that of $A s$; i.e. when $V$ increased, $A s$ decreased; conversely when $V$ decreased, $A s$ increased. An extreme value of the oscillation in $V$ corresponded with an inversion point of the bubble trajectory (marked by $\triangleleft$ in Figure 11), and the one in As corresponded with an inflection point (marked by $<$ in Figure 11). Horizontal bubble velocity increased with increase in concentration up to $150 \mathrm{ppm}$, and decreased with increase in concentration larger than $150 \mathrm{ppm}$.

In order to discuss relation between the zigzagging bubble motion and the interface motion, changes of curvatures $\kappa_{R}$ and $\kappa_{L}$ at right and left edges of the bubble with time are shown in Figure 12. The oscillation of $\kappa_{L}$ was larger than that of $\kappa_{R}$ just after launched. After $0.08 \mathrm{~s}$, when the bubble motion changed to a zigzagging trajectory from a linear trajectory, the oscillation of $\kappa_{L}$ damped; while, the oscillation of $\kappa_{R}$ developed. Subsequently, near an inversion point the oscillation of $\kappa_{R}$ damped; while, the oscillation of $\kappa_{L}$ developed. The phase difference was $\pi / 2$. In the case of purified water, a bubble rose with alternately repeated damping and growth of curvature oscillation. The high-frequency component $(67 \mathrm{~Hz})$ of the curvature oscillation corresponded almost with that of velocity oscillation. In the case of $500 \mathrm{ppm}$, an oscillation of $\kappa_{L}$ was observed only for a very short time just after launched. Subsequently, the oscillation was not observed. These phenomena are considered to be owing to damping effect of the surfactant adsorbed on the bubble surface (Tomiyama et al., 2002).

At $150 \mathrm{ppm}$, at which the zigzag trajectory and bubble velocity behaved characteristically, the oscillations of $\kappa_{R}$ and $\kappa_{L}$ changed gradually with bubble rising. Although amplitudes of the high-frequency oscillations were smaller than those in purified water, clear high-frequency oscillations were observed just after launched. Damping of the oscillation of $\kappa_{R}$ began at about $0.18 \mathrm{~s}$ after launched, and that of $\kappa_{L}$ at about $0.26 \mathrm{~s}$ after launched. Since the adsorption amount of 1-pentanol was small at launch, the initial surface oscillation was considered to be not damped by the adsorbed surfactant. The adsorption amount of the surfactant increased gradually during its rising in the solution; accordingly the surface oscillation is considered to be damped by the increased surfactant.

\subsection{Marangoni effect}


In a very low concentration solution of 1-pentanol, an adsorption-concentration gradient is formed on a bubble surface; on the frontal surface of the bubble, the number of the surfactant molecules adsorbed on the surface is very small; on the rear surface, the number is large. The adsorption-concentration gradient induces Marangoni convection (surface convection on an interface). The Marangoni convection occurs in the direction from the higher concentration of the surface contamination to the lower (see Figure 13).

Hence, the bubble velocities $\bar{w}$ and $\bar{V}$ are deaccelerated by the Marangoni convection. In the solution of 500 ppm, even in the phase of bubble formation, a sufficient number of surfactant molecules are adsorbed on the bubble surface. Indeed, the surface oscillation was significantly damped; in a contaminated bubble, initial surface oscillation is damped (Tomiyama, et al., 2002). The bubble in the $500 \mathrm{ppm}$ solution reduced its own velocities due to Marangoni convection. From the viewpoint of a molecular scale, surfactant molecules reiterate adsorption and desorption on the interface. The rates of adsorption and desorption are important. In the solution of $150 \mathrm{ppm}$, the surface oscillation still remained. The surface contamination by 1-pentanol in the $150 \mathrm{ppm}$ solution is so low; hence the surface oscillation is not damped. The instability of the adsorption-concentration gradient on the bubble interface (i.e. the instability of Marangoni convection) and the asymmetricity of bubble surface oscillation induced decrease in $\bar{w}$ and increase in $|\bar{u}|$. To discuss the increase in $|\bar{u}|$ in $150 \mathrm{ppm}$ solution, the information about the surrounding liquid motion of the bubble is required.

\subsection{Motion of surrounding liquid}

Isograms of vorticity calculated from PIV results are plotted in Figure 14 (solid lines: positive, broken lines: negative, an arrow: trajectory of bubble motion, an interval of the isolines: $16[1 / \mathrm{s}])$. The numbersymbols represent values of the outmost isolines. Figure 14 (a) shows vorticity isograms in purified water near the transition point from vertically straight motion to zigzag motion; (b) shows them near the second inversion point. In Figure 14 (a), positive and intense vorticity was observed at the lower-left of the bubble; hence the vorticity profile at rear of the bubble was unsymmetrical. Although bubble-wake structure and bubble shape were symmetric during the vertically straight ascent motion of the bubbles; near the transition point the symmetry was not kept. Subsequently vortex shedding occurred; as a result the bubble changed its direction of the motion. Coupling these results with the surface motion described in section 3.4, the vortex shedding is considered to relate closely to the surface motion of the bubble. In every 1-pentanol concentration, after vortex shedding, oscillation of the curvature of the bubble edge at the outside of the trajectory is larger than that of the opposite side. The vortex observed in the rear of the bubble (marked by broken circle) is considered to be a part of well-known hairpin-like vortex (Brüker, 1999; Lunde and Perkins, 1997). Figures 14 (c) and (d) show vorticity isograms near the first transition point in 150 and 500 ppm solutions of 1-pentanol, respectively. In both the case, vortex shedding was observed at the outside of the trajectory 
as well as in the case of purified water. However, intensity of the vorticity observed at the rear (i.e. far-wake region) of the bubble is different among these figures.

The intensity in the $150 \mathrm{ppm}$ solution is larger than those of the others (i.e. purified water and 500 ppm solution). Intensity in 500 ppm solution is slightly smaller than that in purified water. The changes in intensity of the vorticity are considered to be caused by changes of hydrodynamic properties of the gas-liquid interface due to adsorption of the surfactants. Takagi at al. (2003) reported that the wakes of contaminated bubbles are intensively influenced by their interface conditions, using computational method; i.e. non-slip or slip. The surfactant distribution on the interface is not uniform on the interface: on the frontal interface of the bubble, the slip condition is approved because of non- or negligible adsorption of the surfactant; on the rear interface of the bubble, the non-slip condition is approved because of much adsorption of the surfactant. In the $150 \mathrm{ppm}$ solution, a separation point is considered to fluctuate along with fluctuation of a boundary of non-slip (i.e. much contaminated interface) and slip (non- or negligible adsorption of surfactant) condition; hence the intensity of the vorticity tends to become larger. On the other hand, in the $500 \mathrm{ppm}$ solution, an enough amount of surfactant is adsorbed; as a result smooth distribution of surfactant might exist; therefore the intensity of vorticity tends to become smaller. Furthermore, initial shape deformation at $500 \mathrm{ppm}$ solution is smaller than those in other concentrations, due to the adsorption of the surfactant; consequently surface free energy of the bubble becomes smaller. A scenario to explain the surfactant effects on the bubble zigzagging motion and the surrounding liquid motion is proposed as follows:

1) A bubble in the range of the present study is forced to zigzag move by vortex shedding,

2) The vortex shedding as well as the intensity and size of the vorticity at rear region of a bubble depend on condition of the contaminated interface,

3) The condition of the contaminated interface depends on surfactant concentration and adsorption/desorption rate,

4) The surfactant concentration on the bubble interface depends on the surrounding liquid motion and bulk concentration of the surfactant,

5) These interactions among the bubble motion, its surrounding liquid motion and surfactant adsorption/desorption play important roles to both the bubble motion and surrounding liquid motion.

\section{CONCLUSION}

The liquid-phase velocities around a zigzagging ascent bubble, the centre-of-gravity and surface motion of the bubble were discussed in purified water and 1-pentanol solutions using recursive cross-correlation algorithm and a high-speed video camera. The analysis code of a newly developed PIV system showed the high performance in measuring velocities of wide dynamic range. At first, the authors demonstrate the performance of the recursive cross-correlation PIV algorithm compared with the results obtained by conventional type of PIV algorithm using the standard PIV images of wide dynamic range presented by The Visualization Society of Japan. 
At second, the developed system was applied to measure velocity field around a zigzagging ascent bubble, and its surface motion simultaneously. At third, surfactant effects on the surrounding liquid motion (i.e. intensity of the vorticity, size of the vorticity and the structure of a hairpin-like vortex) were discussed as well as those on centre-ofgravity and surface motion of the bubble. In this study, a very small amount of surfactant (1-pentanol) solutions were examined. Although the bulk surface tension was almost the same as that of purified water, the bubble motion and the surrounding liquid motion in the solutions (i.e. contaminated system) were very different from those in purified water (i.e. purified system). A critical concentration of a surfactant was found out. At the critical concentration, the intensity and size of the vorticity becomes the largest. From the consideration coupling the asymmetric surface motion of the bubble and the surrounding liquid motion, it has been found out that the Marangoni stress owing to a concentration gradient of the adsorbed surfactant on the bubble surface plays a great role.

\section{NOMENCLATURE}

$A_{n}$ : coefficient of FFT expression of a bubble contour [-]

$B_{n}$ : coefficient of FFT expression of a bubble contour [-]

$A_{S}:$ aspect ratio of a bubble [-]

$C_{c c}$ : cross-correlation coefficient [-]

$D_{I}$ : major diameter of a bubble [m]

$D_{2}$ : minor diameter of a bubble [m]

$D_{e q}$ : equivalent diameter of a bubble $[\mathrm{m}]$

$f$ : brightness distribution function [-]

$F$ : power spectra of $f[-]$

$g$ : brightness distribution function [-]

$G$ : power spectra of $g[-]$

$n$ : mode order

$r$ : polar coordinate [-]

$S_{N R}: \mathrm{S} / \mathrm{N}$ ratio [-]

$t$ : time [s]

$\Delta t$ : time interval [s]

$|\bar{u}|:$ average of horizontal velocity component of a bubble $[\mathrm{m} / \mathrm{s}]$

$V$ : bubble composition velocity $[\mathrm{m} / \mathrm{s}]$

$\bar{V}:$ average of $V[\mathrm{~m} / \mathrm{s}]$

$w$ : vertical velocity component of a bubble $[\mathrm{m} / \mathrm{s}]$ 
$\bar{w}:$ average of $w[\mathrm{~m} / \mathrm{s}]$

$x$ : coordinate in an interrogation area [-]

$\Delta x$ : displacement $[\mathrm{m}]$

$\Delta X$ : interval of velocity point of PIV [-]

$y$ : coordinate in an interrogation area [-]

$\Delta y$ : displacement $[\mathrm{m}]$

$\Delta Z$ : interval of velocity point of PIV

Greek letter

$\Gamma$ : circulation $[-]$

$\xi$ : coordinate in an interrogation area [-]

$\eta$ : coordinate in an interrogation area [-]

$\theta$ : polar coordinate $[\mathrm{rad}]$

$\kappa$ : bubble-edge curvature normalized by equivalent diameter of a bubble [-]

$\omega$ : vorticity [-]

Subscript

1: the first phase in recursive PIV or $\theta$ at major diameter

L: left

$\mathrm{R}$ : right

\section{REFERENCES}

Adrian, R. J., (1991), "Particle-imaging technique for experimental fluid mechanics", Annual Review of Fluid Mechanics, 23, 261-304.

Brüker, C., (1999), "Structure and dynamics of the wake of bubbles and its relevance for bubble interaction", Physics of Fluids, 11(7), 781-1796.

Duineveld, P.C., (1995), “The rise velocity and shape of bubbles in pure water at high Reynolds number”, J. Fluid Mech., 292, 325-332.

Ellingsen, K. and F. Risso, (2001), “On the rise of an ellipsoidal bubble in water”, J. Fluid Mech., 440, 235-268.

Fan, L. S. and K. Tsuchiya, (1990), "Bubble Wake Dynamics in Liquids and Liquid-solid Suspensions", Butterworth-Heinemann.

Fujiwara, A., Y. Danmoto, K. Hishida and M. Maeda, (2004), "Bubble deformation and flow structure measured by double shadow images and PIV/LIF", Experiments in Fluids, 36(1), 157-165 
Hart, D.P., (2000), “PIV error correction,” Experiments in Fluids, 29(1), 13-22.

Lunde, K. and R. Perkins, (1997), “Observations on wakes behind spheroidal bubbles and particles”, ASME-FED Summer Meeting, paper No. FEDSM97-3530.

Miyamoto, Y. and T. Saito, (2005), "Relationship between Interface Motion of an Isolated Bubble and Its Zigzagging Motion of the Centre of Gravity", Transactions of the Japan Society of Mechanical Engineers, Series $B, 71(705)$, 1307-1313, (in Japanese).

Sakakibara, K., M. Yamada, Y. Miyamoto, and T. Saito, (2006), "Measurement of the Surrounding Liquid Motion of a Single Rising Bubble Using a Dual-camera PIV System", Proceedings of the 5th International Symposium on Measurement Techniques for Multiphase Flows, 2, 1071- 76.

Sakakibara, K., M. Yamada, Y. Miyamoto, and T. Saito, (2007), "Measurement of the Surrounding Liquid Motion of a Single Rising Bubble Using a Dual-camera PIV System”, Flow measurement and Instrumentation, 18(5-6), 211-215.

Sanada, T., T. Watanabe, and T. Fukano, (2006), "Interaction and Coalescence of Bubbles in Stagnant Liquid", Multiphase Science and Technology, 2(18), 155-174.

Takagi, S., T. Uda, Y. Watanabe and Y. Matsumoto, (2003), "Behaviour of a Rising Bubble in Water with Surfactant Dissolution (1st Report, Steady Behaviour)", Transactions of the Japan Society of Mechanical Engineers, Series B, 69(686), 2192- 2199, (in Japanese).

Tomiyama, A., G. P. Celate, S. Hosokawa and S. Yosida, (2002), "Terminal velocity of single bubbles in surface tension force dominant regime", International Journal of Multiphase Flow, 28, 1497-1519.

Westerweel, J., D. Dabiri and M. Gharib, (1997), “The effect of a discrete window offset on the accuracy of crosscorrelation analysis of digital PIV recordings", Experiments in Fluids, 23(1), 20-28.

Wu, M. and M. Gharib, (2002), "Experimental studies on the shape and path of small air bubbles rising in clean water", Physics of Fluids, 14(7), L49-L52. 


\section{List of figures and tables}

Figure 1: Schematic of experimental setup.

Figure 2: Schematic of bubble launching device equipped with an audio speaker.

Table 1: Table 1: Property of VSJ-PIV standard images. (2 dimensional wall shear flow, STD)

Figure 3: A set of PIV standard images presented by The Visualization Society of Japan.

(a) at $t=t_{1}$, (b) at $t=t_{1}+\Delta t$

Figure 4: Outline of conventional cross-correlation PIV.

Figure 5: Outline of recursive cross-correlation PIV.

Figure 6: Results of recursive cross-correlation PIV. (PIV standard images by The Visualization Society of Japan)
(a) $64 \times 64$ pixels,
(b) $32 \times 32$ pixels,
(c) $16 \times 16$ pixels,
(d) $8 \times 8$ pixels

Figure 7: Comparison of velocity distribution and contour maps of $C c c$ and signal intensity $\left(1-1 / S_{N R}\right)$ between conventional cross-correlation PIV and recursive cross-correlation PIV. (Interrogation size: $16 \times 16$ pixels).

(a) $C_{C C}$ (conventional), (b) $C_{C C}$ (recursive), (c) Signal intensity (conventional), (d) Signal intensity (recursive)

Figure 8: Comparison of velocity field between true values and measured values via recursive PIV. Interrogation size: $8 \times 8$ pixels.

(a) True, (b) Measured by recursive PIV

Figure 9: Pre-process for PIV and bubble motion analysis.
(a) Original image,
(b) Particle image,
(c) Bubble image

Table 2: Bubble properties.

Figure 10: Verification of reproducibility of bubble shape, and orientation, and bubble trajectory.
(a) A typical snapshot of a bubble,
(b) A contour of a bubble,
(c) Superimposed contours of 100 bubbles,
(d) Superimposition of (b) and (c)

Figure 11: Bubble trajectory, velocity $V$ and Aspect ratio $A s$ under several surfactant concentrations (1-pentanol).
(a) Purified water,
(b) $150 \mathrm{ppm}$,
(c) $500 \mathrm{ppm}$

Table 3: Relation between bubble motion and 1-pentanol concentration.

Figure 12: Bubble trajectory and curvature $\kappa_{R}$, and $\kappa_{L}$.
(a) Purified water,
(b) $150 \mathrm{ppm}$,
(c) $500 \mathrm{ppm}$

Figure 13: Pattern diagram of relation between surfactant adsorption and Marangoni convection.

Figure 14: Vorticity profile of the surrounding liquid of a bubble.
(a) Purified water $(\mathrm{t}=0.25 \mathrm{~s})$,
(b) Purified water $(\mathrm{t}=0.73 \mathrm{~s})$,
(c) $150 \mathrm{ppm}(\mathrm{t}=0.15 \mathrm{~s})$,
(d) $500 \mathrm{ppm}(\mathrm{t}=0.12 \mathrm{~s})$ 


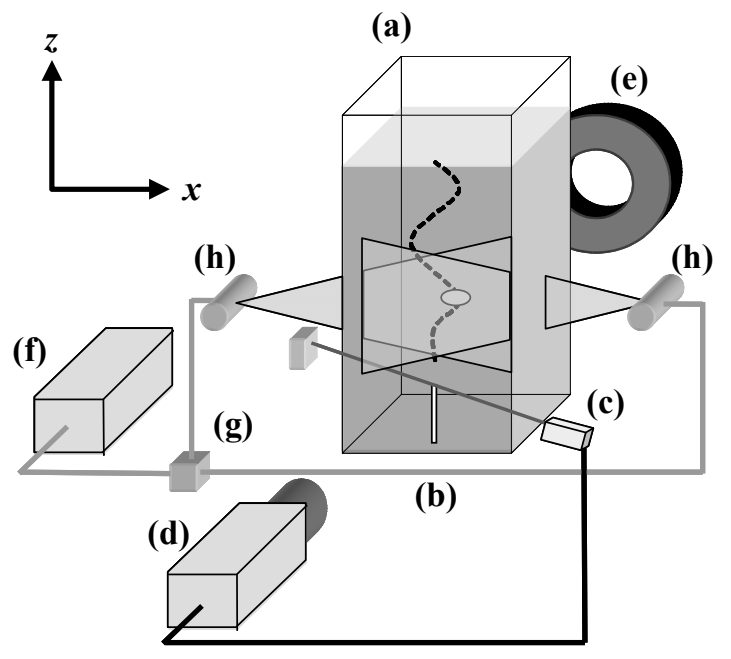

(a) Water vessel, (b) Needle, (c) Laser sensor, (d) Camera,

(e) Continuous LED, (f) CW laser, (g) Half mirror, (h) Rod lens

Figure 1: Schematic of experimental setup. 


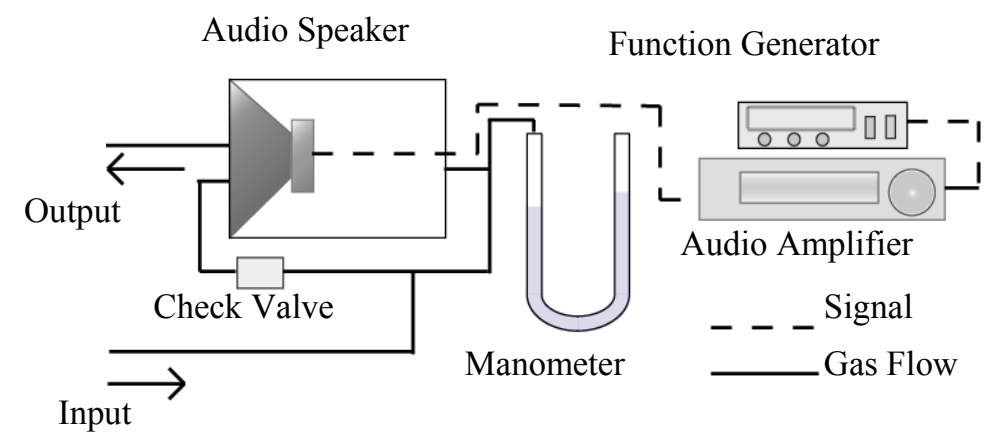

Figure 2: Schematic of bubble launching device equipped with an audio speaker. 
Table 1: Property of VSJ-PIV standard images.

(2 dimensional wall shear flow, STD)

\begin{tabular}{ll}
\hline Image size & $256 \times 256$ pixels \\
\hline Area & $100 \times 100 \mathrm{~mm}$ \\
\hline Laser thickness & $20 \mathrm{~mm}$ \\
\hline Time interval & $33 \mathrm{~ms}$ \\
\hline Mean velocity & $89 \mathrm{~mm} / \mathrm{s}$ \\
\hline Particle displacement & 7.5 pixel/interval \\
Mean & $3.0 \mathrm{pixel} /$ interval \\
Standard deviation & $15.0 \mathrm{pixel} /$ interval \\
maximum & \\
\hline Out of plane motion & $0.017-/$ interval \\
Mean & $0.012-/$ interval \\
Standard deviation & $1.4-$ \\
\hline Image vorticity & $4000-$ \\
\hline Number of particles & \\
\hline Particle diameter & $5.0 \mathrm{pixels}$ \\
Mean & 1.4 pixels
\end{tabular}




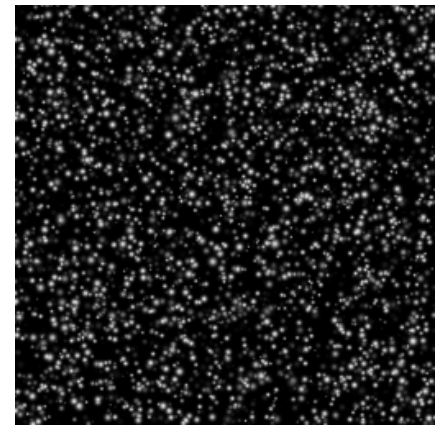

(a) at $t=t_{1}$

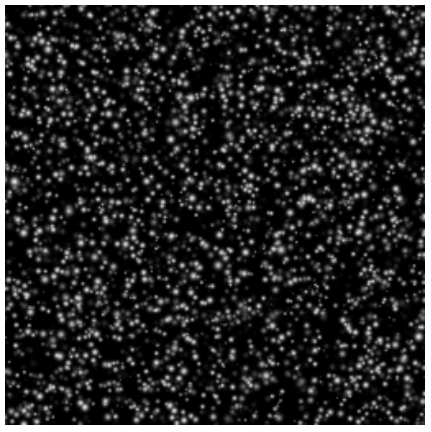

(b) at $t=t_{1}+\Delta t$

Figure 3: A set of PIV standard images presented by The Visualization Society of Japan. 

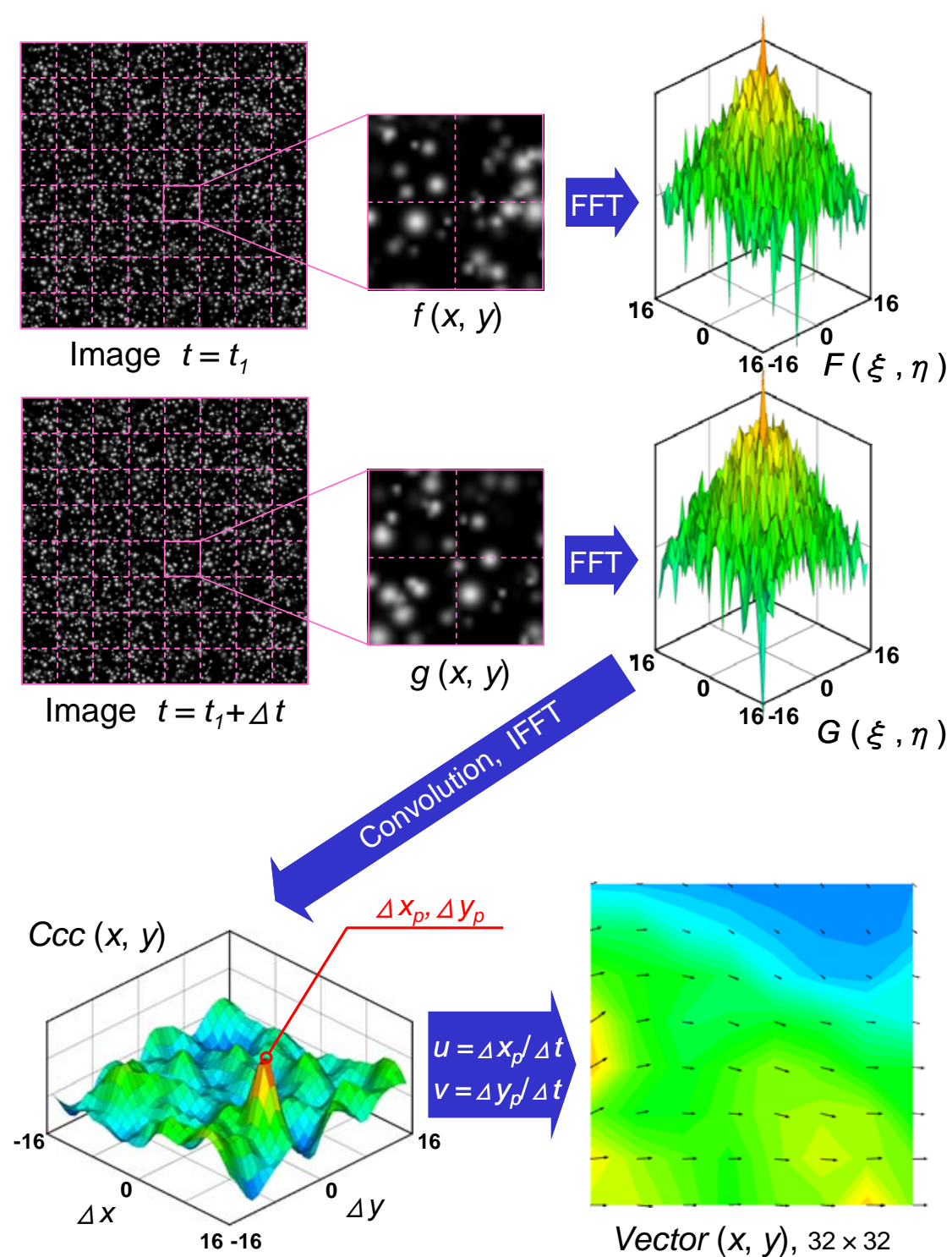

$\operatorname{Vector}(x, y), 32 \times 32$

Figure 4: Outline of conventional cross-correlation PIV. 


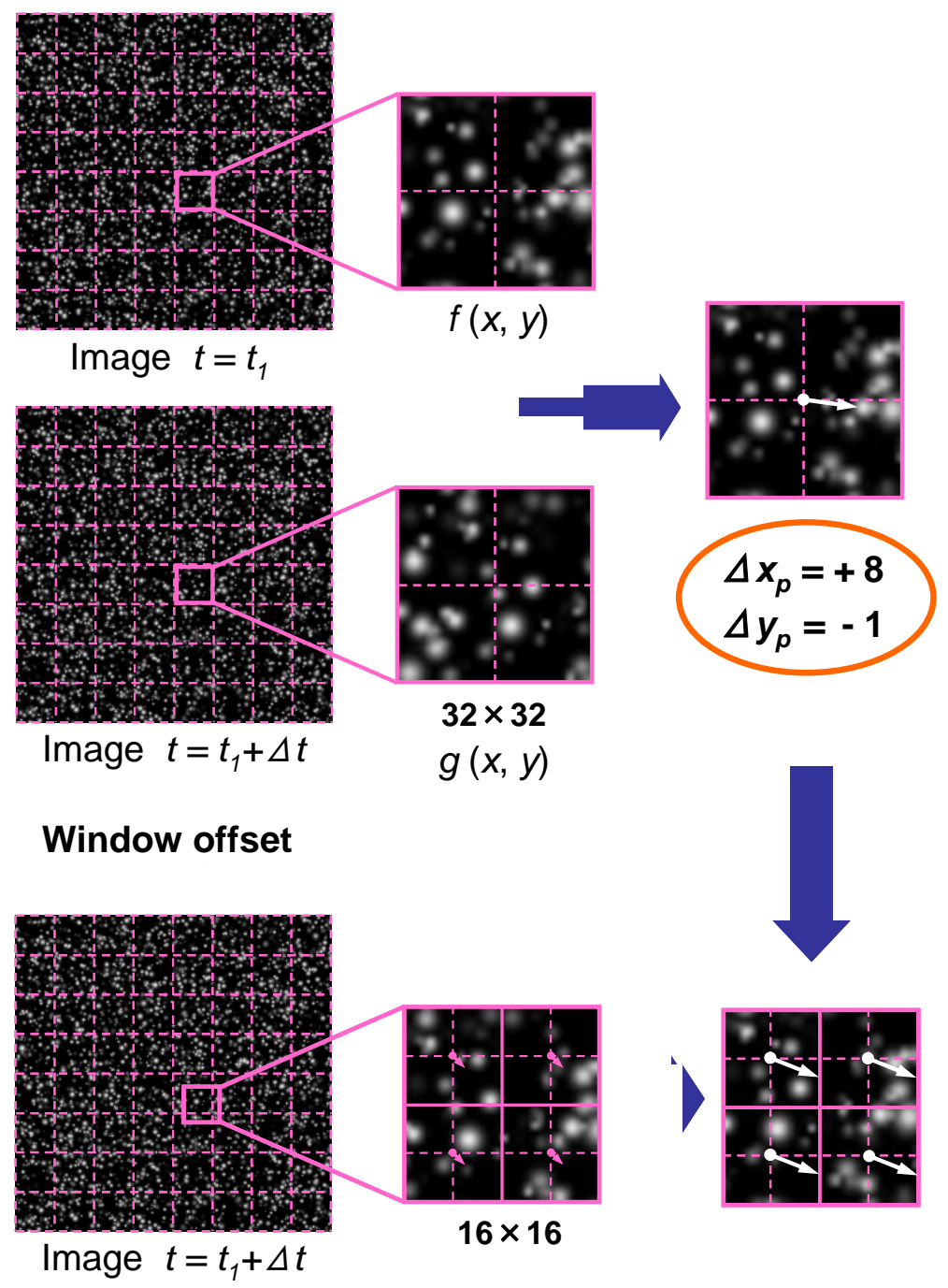

Figure 5: Outline of recursive cross-correlation PIV. 


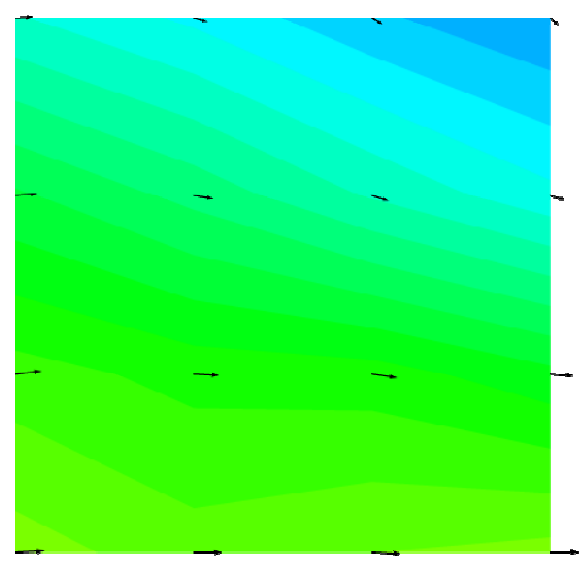

(a) $64 \times 64$ pixels

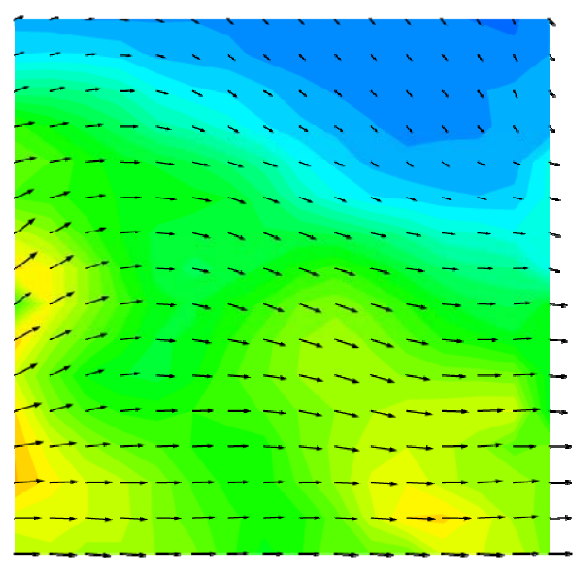

(c) $16 \times 16$ pixels

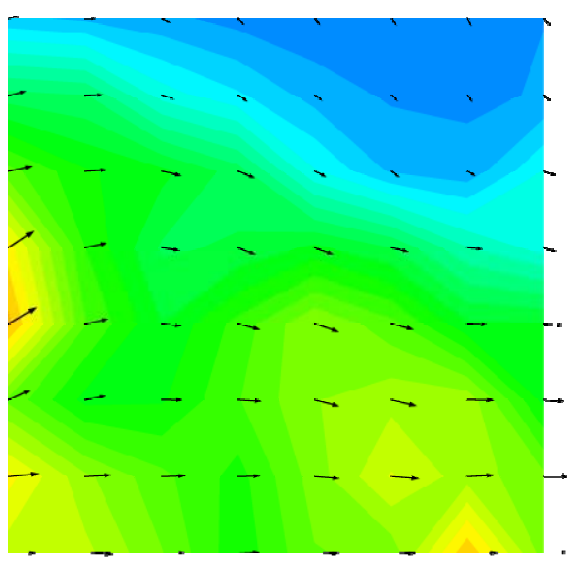

(b) $32 \times 32$ pixels

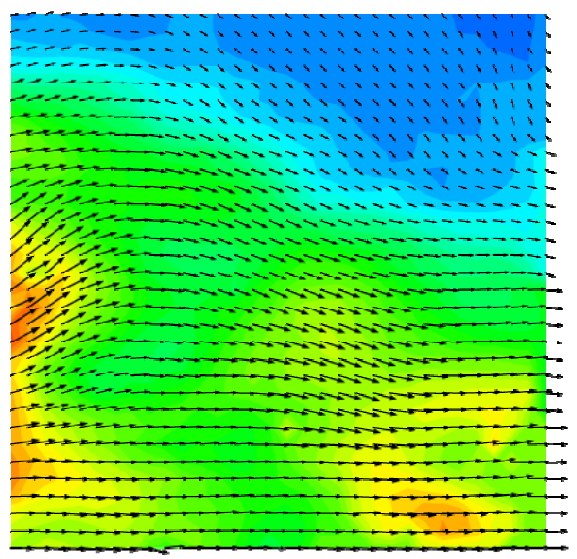

(d) $8 \times 8$ pixels

Figure 6: Results of recursive cross-correlation PIV. (PIV standard images by The Visualization Society of Japan) 


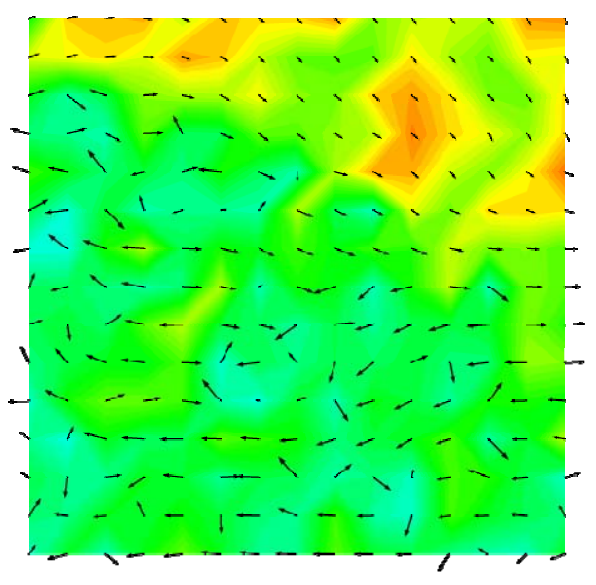

(a) $C_{C C}$ (conventional)

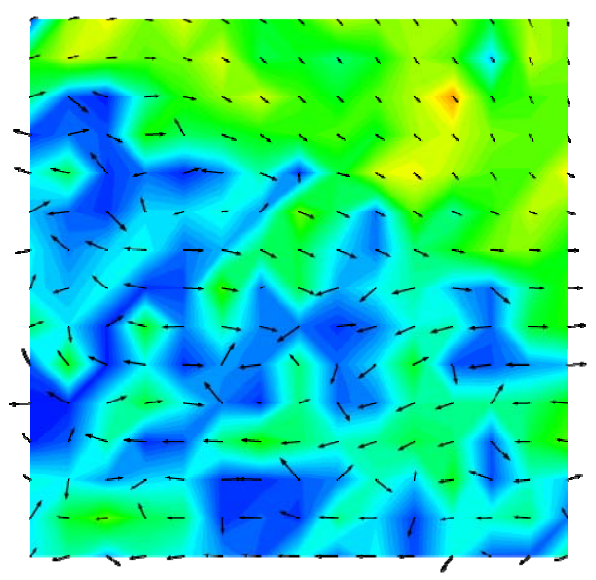

(c) Signal intensity (conventional)

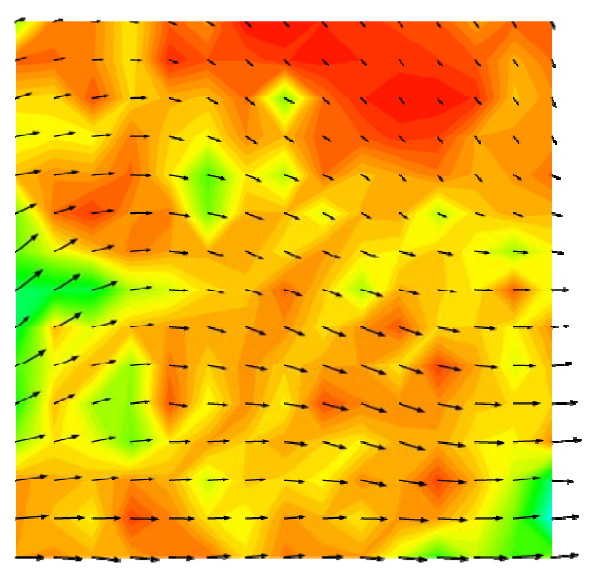

(b) $C_{C C}$ (recursive)

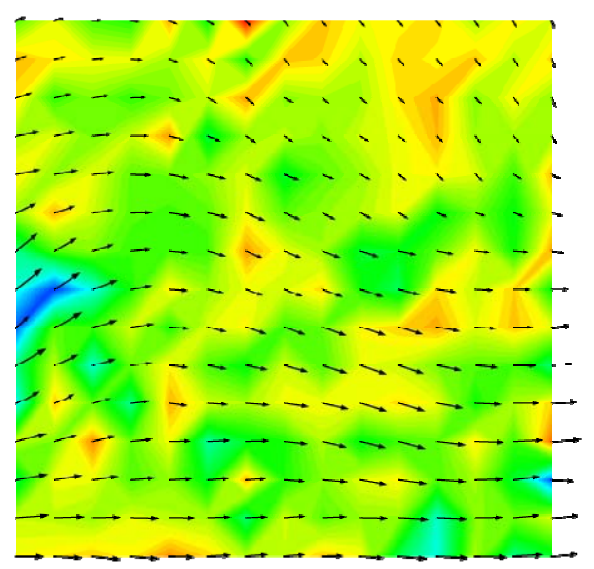

(d) Signal intensity (recursive)

Figure 7: Comparison of velocity distribution and contour maps of $C c c$ and signal intensity $\left(1-1 / S_{N R}\right)$ between conventional cross-correlation PIV and recursive cross-correlation PIV. (Interrogation size: $16 \times 16$ pixels). 


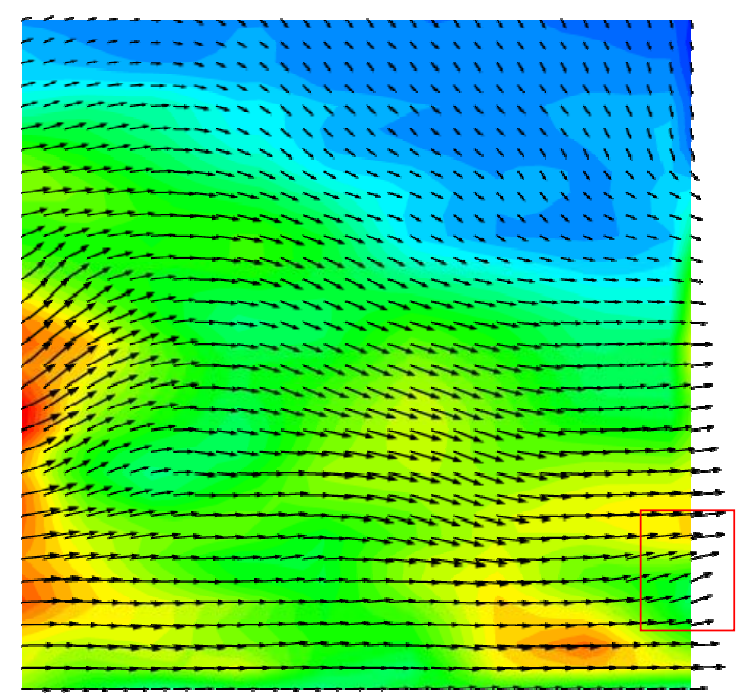

(a) True

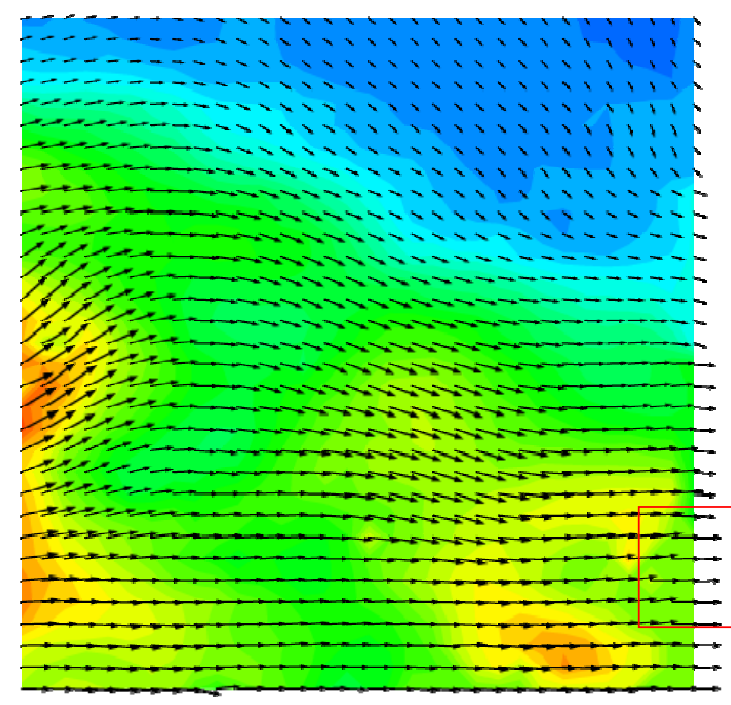

(b) Measured by recursive PIV

Figure 8: Comparison of velocity field between true values and measured values via recursive PIV. Interrogation size: $8 \times 8$ pixels. 


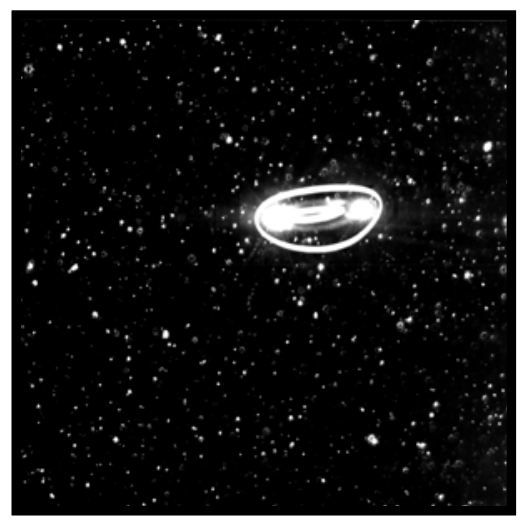

(a) Original image

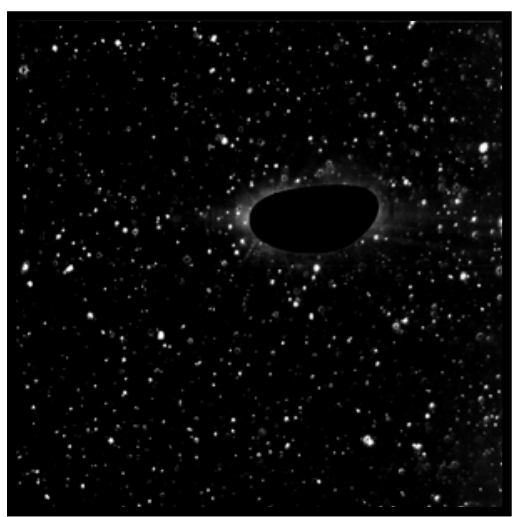

(b) Particle image

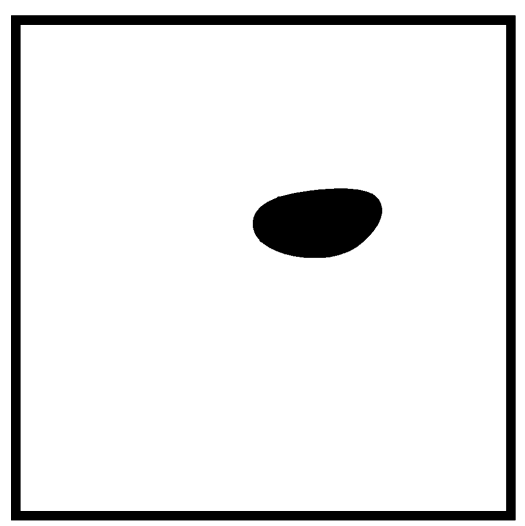

(c) Bubble image

Figure 9: Pre-process for PIV and bubble motion analysis. 
Table 2: Bubble properties.

\begin{tabular}{|c|c|c|c|c|c|}
\hline $\begin{array}{c}\text { Concentration } \\
{[\mathrm{ppm}]}\end{array}$ & $\begin{array}{c}D_{e q} \\
{[\mathrm{~mm}]}\end{array}$ & $\begin{array}{c}\sigma \\
{[\mathrm{mN} / \mathrm{m}]}\end{array}$ & $\begin{array}{c}R e \\
{[-]}\end{array}$ & $\begin{array}{c}\text { We } \\
{[-]}\end{array}$ & $\begin{array}{c}\text { Eo } \\
{[-]}\end{array}$ \\
\hline 0 & 2.89 & 71.9 & 855 & 3.5 & 1.13 \\
\hline 150 & 2.88 & 71.5 & 820 & 3.3 & 1.13 \\
\hline 500 & 2.83 & 69.9 & 645 & 2.1 & 1.12 \\
\hline
\end{tabular}

$D_{e q}$ : equivalent diameter of bubble, g: gravity acceleration,

$V$ : bubble composition velocity, $\rho_{L}$ : density of liquid, $\sigma$ : surface tension

Reynolds number: $\quad R e=\frac{\rho V D_{e q}}{\mu}$

Weber number: $\quad W e=\frac{\rho V^{2} D_{e q}}{\sigma}$

Eötvös Number: $\quad E o=\frac{g \rho_{L} D_{e q}}{\sigma}$ 


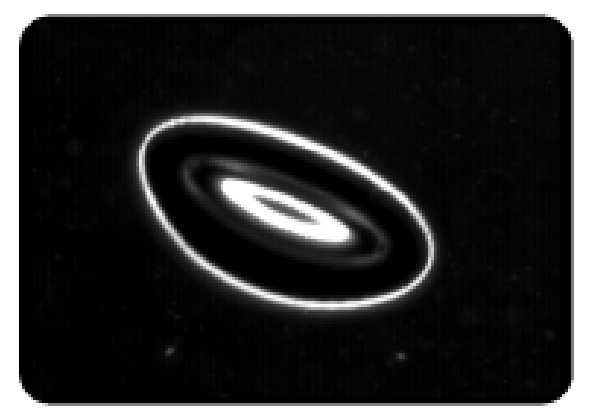

(a) A typical snapshot of a bubble.

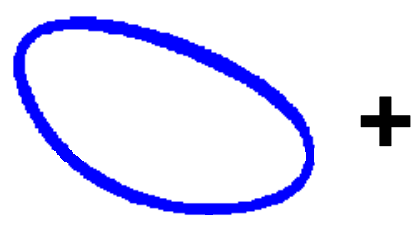

(b) A contour of a bubble.

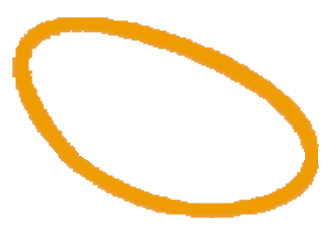

(c) Superimposed contours of 100 bubbles.

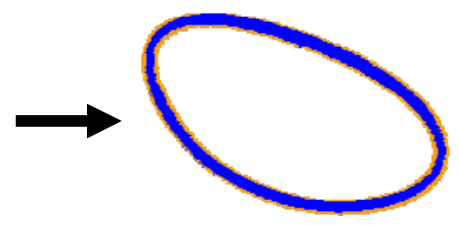

(d) Superimposition of (b) and (c).

Figure 10: Verification of reproducibility of bubble shape, and orientation, and bubble trajectory. 


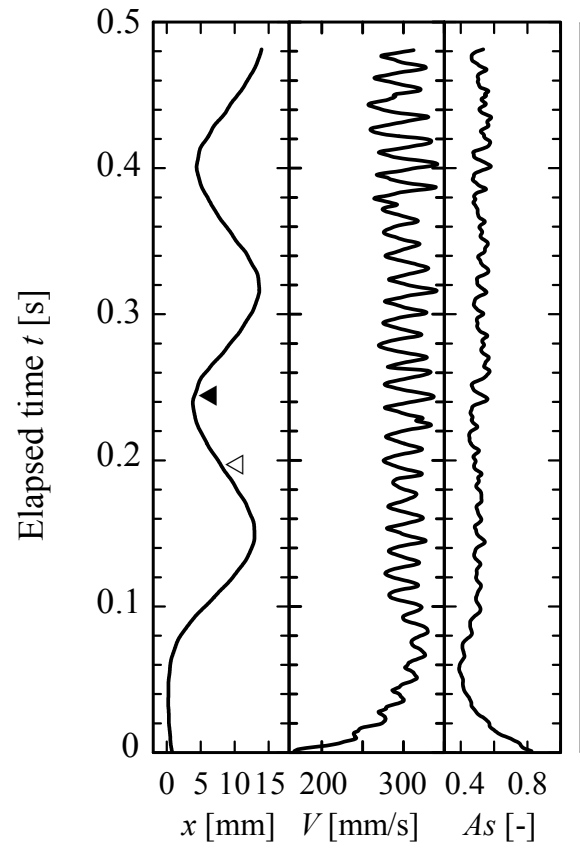

(a) Purified water

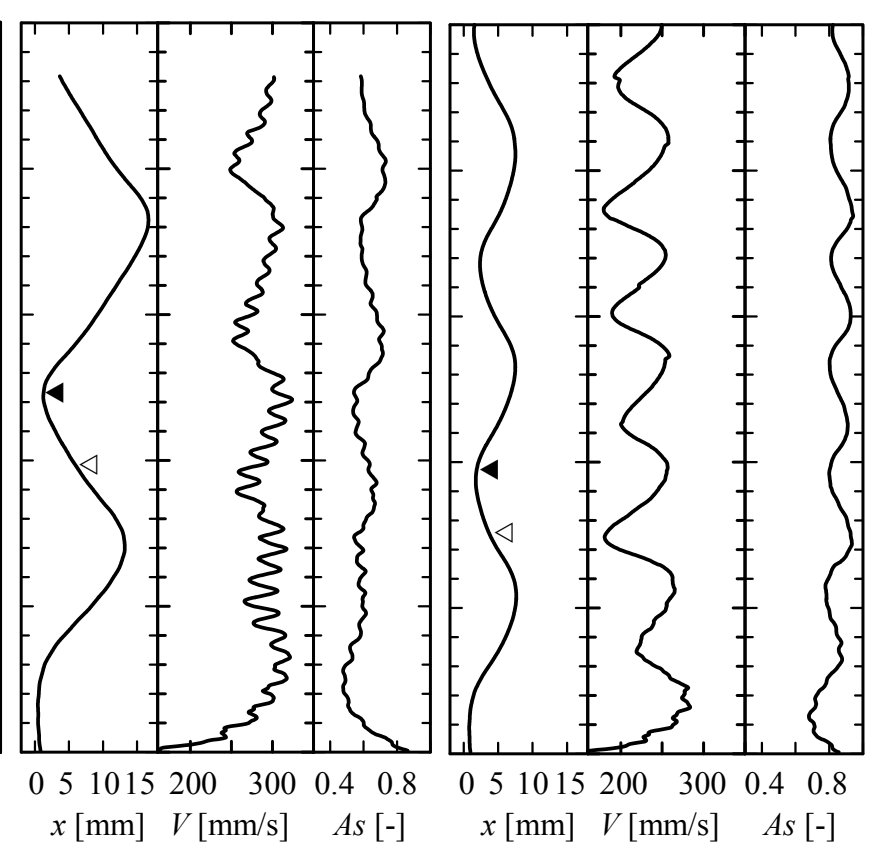

(b) $150 \mathrm{ppm}$

(c) $500 \mathrm{ppm}$

Figure 11: Bubble trajectory, velocity $V$ and Aspect ratio $A s$ under several surfactant concentrations (1-pentanol). 
Table 3: Relation between bubble motion and 1-pentanol concentration.

\begin{tabular}{|c|c|c|c|c|c|c|}
\hline $\begin{array}{c}D_{e q} \\
{[\mathrm{~mm}]}\end{array}$ & $\begin{array}{c}\text { Concentration } \\
{[\mathrm{ppm}]}\end{array}$ & $\begin{array}{c}A_{B z} \\
{[\mathrm{~mm}]}\end{array}$ & $\begin{array}{c}f_{B z} \\
{[\mathrm{~Hz}]}\end{array}$ & $\begin{array}{c}|\bar{u}| \\
{[\mathrm{mm} / \mathrm{s}]}\end{array}$ & $\begin{array}{c}\bar{w} \\
{[\mathrm{~mm} / \mathrm{s}]}\end{array}$ & $\begin{array}{c}\bar{V} \\
{[\mathrm{~mm} / \mathrm{s}]}\end{array}$ \\
\hline 2.89 & 0 & 9.3 & 5.9 & 106 & 270 & 297 \\
\hline 2.88 & 150 & 13.6 & 4.4 & 116 & 254 & 286 \\
\hline 2.83 & 500 & 5.6 & 6.5 & 71 & 213 & 229 \\
\hline
\end{tabular}




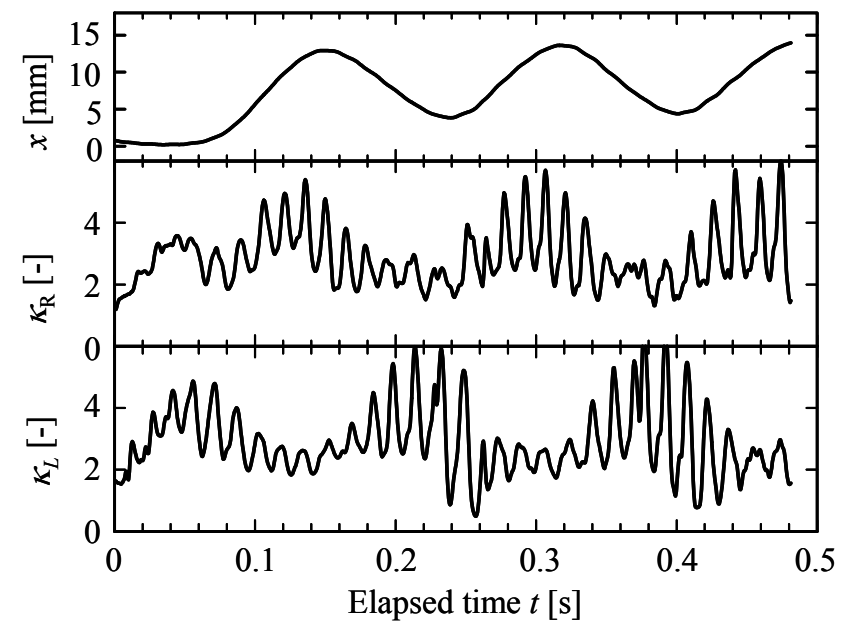

(a) Purified water

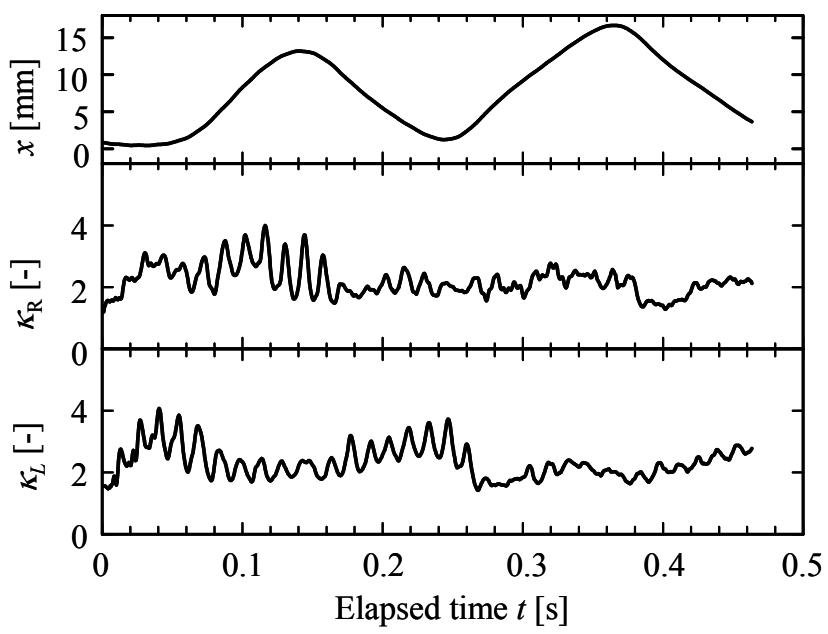

(b) $150 \mathrm{ppm}$

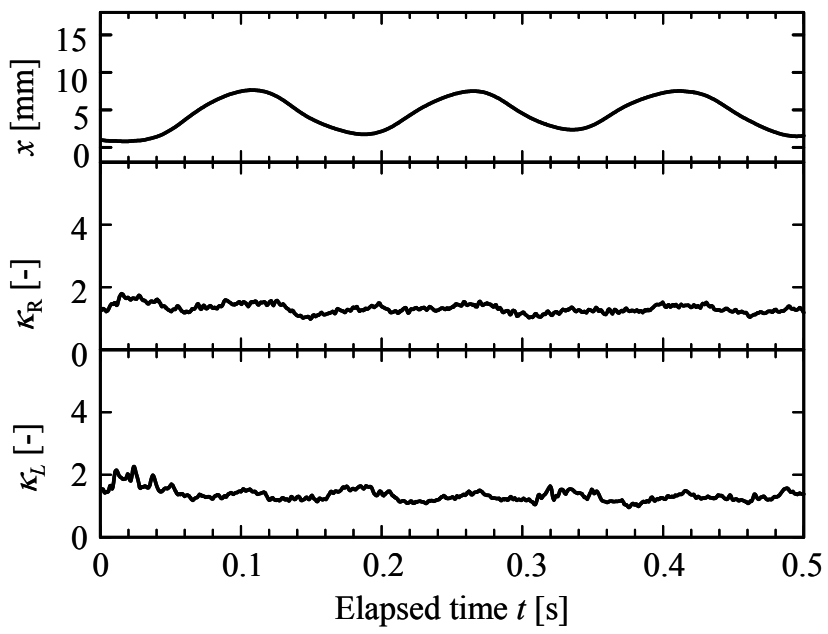

(c) $500 \mathrm{ppm}$

Figure 12: Bubble trajectory and curvature $\kappa_{R}$, and $\kappa_{L}$. 


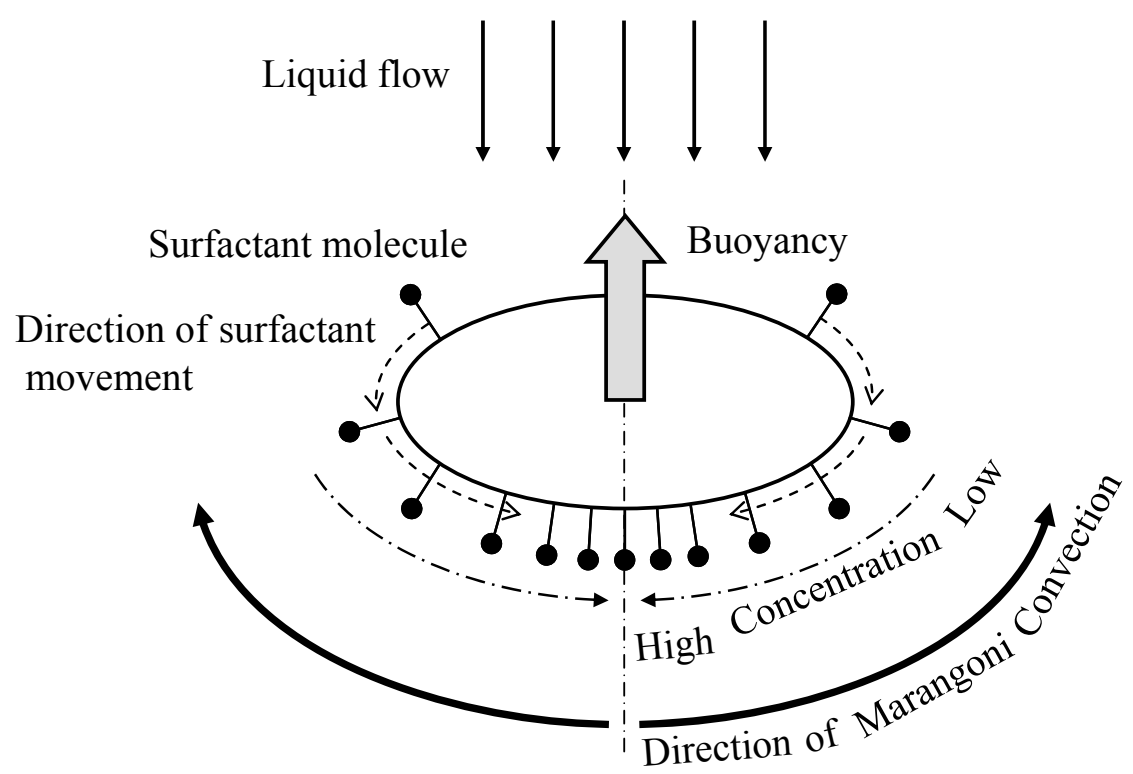

Figure 13: Pattern diagram of relation between surfactant adsorption and Marangoni convection. 


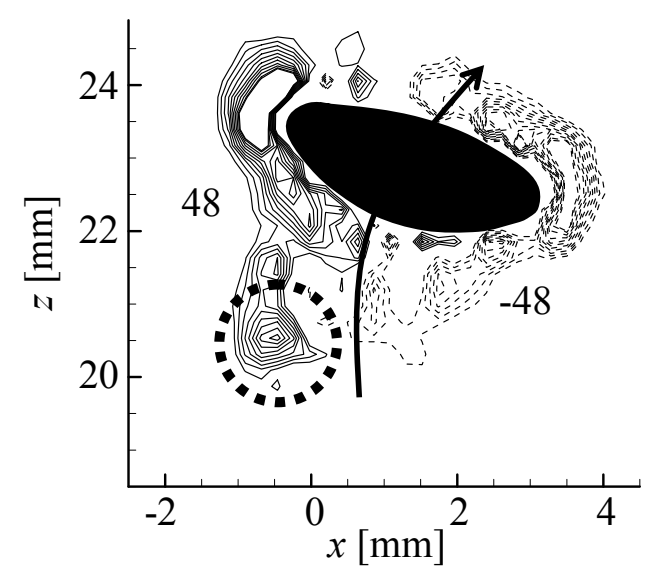

(a) Purified water $(t=0.25 \mathrm{~s})$

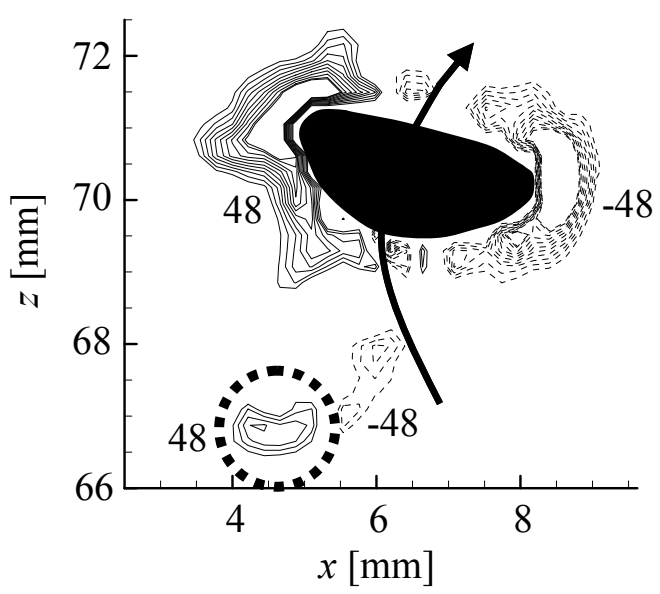

(b) Purified water $(t=0.73 \mathrm{~s})$

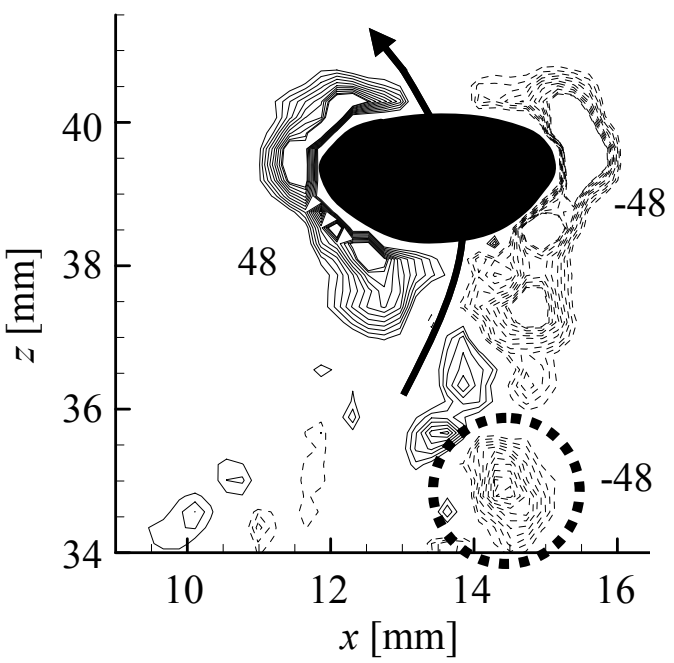

(c) $150 \mathrm{ppm}(t=0.15 \mathrm{~s})$

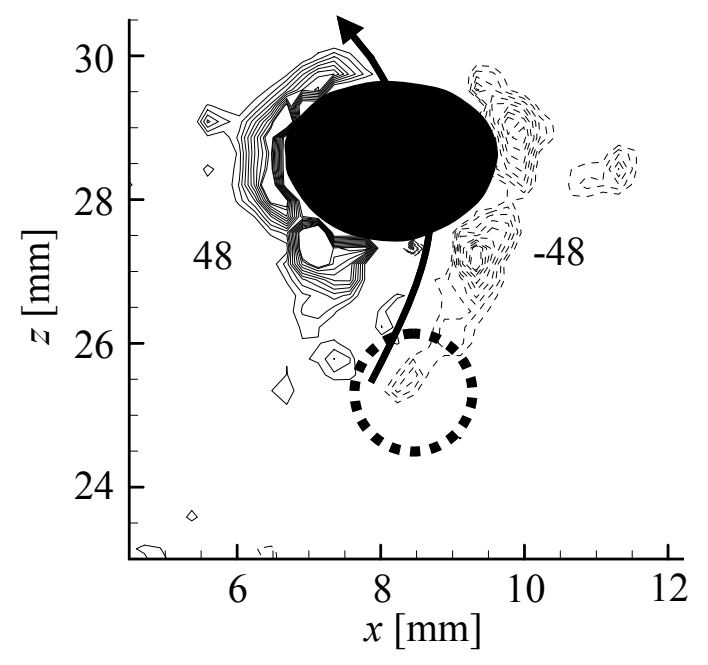

(d) $500 \mathrm{ppm}(t=0.12 \mathrm{~s})$

Figure 14: Vorticity profile of the surrounding liquid of a bubble. 